\title{
III-V Quantum Dots in Dielectrics Made by Ion Implantation and Flash Lamp Annealing
}

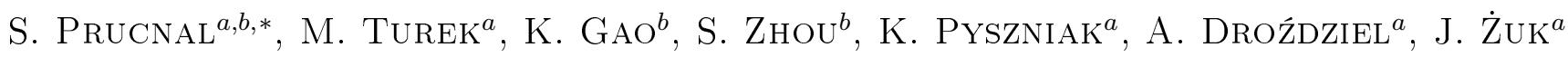 \\ AND W. SKORUPA ${ }^{b}$ \\ ${ }^{a}$ Maria Curie-Skłodowska University, pl. M. Curie-Skłodowskiej 1, 20-031 Lublin, Poland \\ ${ }^{b}$ Institute of Ion Beam Physics and Materials Research, Forschungszentrum Dresden-Rossendorf \\ P.O. Box 510119, 01314 Dresden, Germany
}

\begin{abstract}
Different semiconductor nanocrystals synthesized in dielectrics on silicon are very interesting for applications in non-volatile memories and photovoltaics. In this paper we present an overview of microstructural and opto-electronic properties of different III-V quantum dots embedded in $\mathrm{SiO}_{2}$ and $\mathrm{Si}_{3} \mathrm{~N}_{4}$ made by sequential ion implantation and millisecond range flash lamp annealing. It is shown that within $20 \mathrm{~ms}$ post-implantation annealing high quality crystalline III-V quantum dots can be formed in different matrices. Formation of crystalline III-V quantum dots was confirmed by cross-section transmission electron microscopy, photoluminescence and $\mu$-Raman spectroscopy. Flash lamp annealing is essentially a single-flash-single-wafer technique whose main attributes are the ease and control of processing over large wafer batches.
\end{abstract}

DOI: 10.12693/APhysPolA.123.935

PACS: 78.30.Fs, 78.67.Hc, 81.05.Ea, 81.07.Ta, 81.15.Lm

\section{Introduction}

Semiconductor quantum dots of the size in the range of the exciton Bohr radius or smaller are very attractive objects for both research and application, due to their special optical and electrical properties. The III-V semiconductors with a direct band gap are widely used in optoelectronics e.g. as the light emitters operating in the wavelength range from ultraviolet to the infrared. Other applications of III-V compounds include single or tandem solar cells, high electron mobility transistors (HEMTs), and colour displays. Moreover, III-V QDs embedded in dielectrics show great potential for the memory devices application such as EEPROM or FLASH EEPROM with III-V nanocrystals as the information storage elements. For such applications the most attractive are $\mathrm{InN}$ and InAs nanocrystals embedded in the $\mathrm{SiO}_{2}$ matrix (electron affinity $0.9 \mathrm{eV}$ ) with the electron affinity of 5 and $4.9 \mathrm{eV}$, respectively [1].

On the other hand, the compound semiconductors III-V with electron mobility from a few (InP $\left.5400 \mathrm{~cm}^{2} /(\mathrm{V} \mathrm{s})\right)$ up to tens time (InSb $77000 \mathrm{~cm}^{2} /(\mathrm{V} \mathrm{s})$ ) higher than is silicon seems to be the most suitable candidate for better electronic devices performance. The lack of production technique fully integrated with silicon technology is a main problem of wide application of III-V compound semiconductors. The main fabrication methods for such QDs are the porous glass method [2], vapour deposition [3], sol-gel synthesis [4] and sequential ion implantation [5-8]. The QDs can have several

*corresponding author; e-mail: s.prucnal@hzdr.de shapes depending on the manufacturing method, which has strong influence on their optical and electrical properties [9]. A technique alternative to the epitaxial growth of III-V nanostructures is the high fluence ion implantation followed by post-implantation annealing. With this method any kind of compound semiconductors can be formed in any solid substrate [9]. Recently we have shown that the ion implantation combined with millisecond range flash lamp annealing (FLA) can be successfully used for integration of III-V compound semiconductors with silicon technology. Based on our method the high quality single crystalline InAs and InP quantum dots (QDs) were produced on (100) oriented $p$-type silicon wafer $[10,11]$. In consequence, the $n-\mathrm{III}-\mathrm{V} / p-\mathrm{Si}$ heterojunction was obtained.

In this paper we present an overview of different III-V QDs formation in the $\mathrm{SiO}_{2}$ and $\mathrm{Si}_{3} \mathrm{~N}_{4}$ hosts by ion implantation and post-implantation annealing. We have synthesized indium and gallium based QDs such as: InSb, InAs, GaAs, and GaN in both dielectrics. Both the cross-section transmission electron microscope (TEM) and $\mu$-Raman spectroscopy show that the high temperature millisecond range FLA is sufficient to form crystalline III-V QDs in dielectrics. In the case of GaAs QDs, the FLA gives an opportunity to control both the crystallographic orientation and conductivity type of nanodots, while GaN crystals embedded in dielectric are interesting for the ultraviolet light emitters.

\section{Experimental part}

Single crystalline (100) oriented $p$-type silicon wafers were covered by either dry thermal oxide or silicon nitride layer deposited by plasma enhanced chemical vapor deposition (PECVD). The thickness of dielectrics was $100 \mathrm{~nm}$. 
Dielectric layers were sequentially implanted with ions belonging to groups III and V [12-14]. The implantation energies were chosen in such a way that the spatial distribution of implanted elements overlap each other and most of the implanted elements are located either in the middle of dielectric layer or close to the silicon/dielectric interface. After ion implantation the post-implantation annealing was performed. The samples were annealed for $20 \mathrm{~ms}$ in the temperature range $1000-1400^{\circ} \mathrm{C}$ using Flash Lamp System at Helmholtz Zentrum Dresden-Rossendorf with preheating at 600 or $700^{\circ} \mathrm{C}$ for 3 min. The preheating was used to initialize cluster formation of III-V QDs.

\section{TABLE}

Implantation parameters for III-V QDs formation in the $\mathrm{SiO}_{2}$ and $\mathrm{Si}_{3} \mathrm{~N}_{4}$ layer. Given implantation energy provides $R_{\mathrm{p}}$ in the middle of dielectric. In order to form the III-V QDs close to the Si/dielectric interface the implantation energy was increased by $20 \%$.

\begin{tabular}{c|c|c|c|c|c|c|c}
\hline \hline \multicolumn{2}{c|}{ Implanted element } & $\mathrm{In}$ & $\mathrm{As}$ & $\mathrm{Sb}$ & $\mathrm{P}$ & $\mathrm{Ga}$ & $\mathrm{N}$ \\
\hline $\begin{array}{c}\text { implantation } \\
\text { energy [keV] }\end{array}$ & $\mathrm{SiO}_{2}$ & 120 & 100 & 125 & 50 & 100 & 20 \\
\multicolumn{2}{l}{ ion fluence [ion $\left./ \mathrm{Sm}_{3} \mathrm{~N}_{4}\right]$} & 160 & 120 & 170 & 70 & 120 & 30 \\
\hline
\end{tabular}

The ion implantation parameters are summarized in Table. The microstructural and optical properties of III-V QDs were investigated by high-angle annular dark-field scanning TEM (HAADF-STEM) in cross-sectional geometry by means of FEI Titan 80-300 STEM operating at $300 \mathrm{keV}$, Auger spectroscopy, $\mu$-Raman spectroscopy and temperature dependent photoluminescence (TDPL). $\mu$-Raman spectroscopy was performed in a backscattering geometry in the range of 150 to $600 \mathrm{~cm}^{-1}$ using a $532 \mathrm{~nm} \mathrm{Nd}$ :YAG laser. The same type of laser was used for the TDPL. The PL signal was recorded in the temperature range $15-300 \mathrm{~K}$ with a monochromator (Jobin Yvon Triax 320) and a photomultiplier (Hamamatsu H7732-10) in the visible spectrum regions.

\section{Results and discussion}

The microstructural properties of ion implanted and annealed samples were investigated by means of the transmission electron microscope in the cross-section geometry by keeping the electron beam along [011]-zone axis. According to our knowledge the FLA techniques are the most suitable for high quality III-V nanocrystals formation both in dielectrics and silicon matrices. Therefore in this paper we focus only on the flash lamp annealed samples.

Figure 1 shows the cross-section TEM images of different III-V QDs formed in the $\mathrm{SiO}_{2}$ or $\mathrm{Si}_{3} \mathrm{~N}_{4}$ layers. The $\mathrm{InP}, \mathrm{GaAs}$, and InAs QDs embedded into the $\mathrm{SiO}_{2}$ layer are shown in Fig. 1a, b, and d, respectively, while Fig. 1c presents the InAs QDs formed in the silicon nitride layer. We did not find significant differences between both matrices having an influence on the III-V QDs formation process. Location within the dielectrics and the average diameter of the QDs can be easily controlled by varying the ion implantation and annealing parameters. An increase in the ion fluence or annealing temperature in-
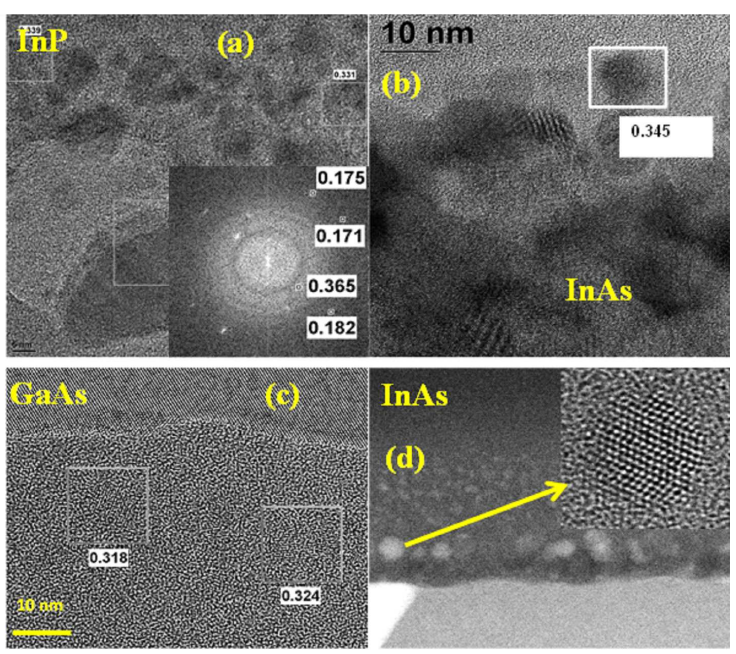

Fig. 1. Cross-sectional TEM images of InP (a), GaAs (c), and InAs (d) nanocrystals in $\mathrm{SiO}_{2}$, and InAs QD in $\mathrm{Si}_{3} \mathrm{~N}_{4}$ (b) obtained from the samples annealed at $1200{ }^{\circ} \mathrm{C}$ for $20 \mathrm{~ms}$ with preheating at $600^{\circ} \mathrm{C}$ for $3 \mathrm{~min}$. The interplanar distances of specific QDs obtained from the electron diffraction pattern are shown as insets.

creases density and an average diameter of nanocrystals, respectively.

Position of the QDs can be controlled by changing the ion energy during implantation i.e. the QDs can be formed in the middle of dielectric (see Fig. 1a and b) or close to the Si/dielectric interface (see Fig. 1c and d) depending on application. Such a result can be obtained only using millisecond range annealing. On the one hand, the thermal budget deposited into the sample is sufficient for the nanocrystals formation. On the other hand, the annealing time is too short for the diffusion activation of the implanted elements. Therefore the ion fluence used in 
our experiments could be around fivefold lower compared to the data which one may find in literature.

The insets in Fig. 1 show the electron diffraction pattern and the interplanar distances for different III-V quantum dots. The obtained values are close to the tabulated ones for specific compound semiconductors. In the case of the indium and phosphorus implanted samples the electron diffraction pattern clearly indicates (111) oriented InP nanocrystals. The interplanar distances measured in the selected area are in the range $0.171-$ $0.175 \mathrm{~nm}$, and are larger than the interplanar distance for bulk InP with $d_{222}=0.169 \mathrm{~nm}$ [15]. The higher value (compared to the bulk InP) of the interplanar distances suggests a small tetragonal distortion of the zinc-blende InP nanocrystals. A similar effect was observed for other III-V QDs annealed at the temperature slightly higher than the melting point of the bulk semiconductor.

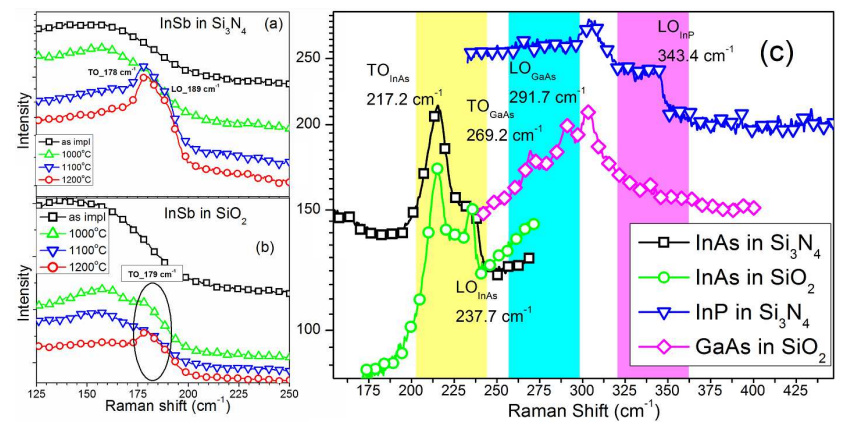

Fig. 2. $\mu$-Raman spectra of In and $\mathrm{Sb}$ implanted and annealed $\mathrm{Si}_{3} \mathrm{~N}_{4}$ (a) and $\mathrm{SiO}_{2}$ layers (b). The millisecond range annealing temperature is indicated in legends. Part (c) shows the $\mu$-Raman spectra obtained from the dielectrics containing InAs, GaAs or InP QDs after annealing at $1200{ }^{\circ} \mathrm{C}$ for $20 \mathrm{~ms}$ with preheating at $600^{\circ} \mathrm{C}$ for $3 \mathrm{~min}$.

In addition, the $\mu$-Raman spectroscopy in the backscattering geometry was performed. Figure $2 \mathrm{a}$ and $\mathrm{b}$ shows the $\mu$-Raman spectra obtained from In and Sb implanted into the oxide and nitride layers and FLA samples, respectively.

The $\mu$-Raman spectra of as implanted sample is shown as well. As can be seen, the flash lamp annealed silicon nitride layer at a temperature higher than $1100{ }^{\circ} \mathrm{C}$ exhibited two peaks at 178 and $189 \mathrm{~cm}^{-1}$, corresponding to the transverse (TO) and longitudinal optical (LO) phonon modes in crystalline InSb [16]. The as implanted sample and annealed up to $1000{ }^{\circ} \mathrm{C}$ shows only one broad peak at around $160 \mathrm{~cm}^{-1}$ which we assign to the amorphous InSb. In the case of the In and Sb implanted silicon dioxide layer some crystalline QDs are formed after annealing at $1000^{\circ} \mathrm{C}$ (see Fig. 2b). Figure 2c shows the $\mu$-Raman spectra obtained from the III-V QDs created in different dielectrics after sequential ion implantation and FLA. As can be seen, the $\mu$-Raman spectra correlate with the cross-section TEM data making the millisecond range FLA treatment suitable for the III-V QDs forma- tion in different dielectrics. We further found that for InAs the process of the QDs formation is more efficient in the nitride layer than in the oxide one, similarly to the InSb QDs. However, independently of the matrix, both TO and LO phonon modes are distinguishable and they are located at 217.2 and $237.7 \mathrm{~cm}^{-1}$, respectively, being close to those of the bulk InAs.

Interestingly, after annealing of the nitride layer at $100{ }^{\circ} \mathrm{C}$ the TO phonon mode was found to be dominant in the spectra, implying that the InAs QDs are mainly oriented along the (111) plane. As the annealing temperature was increased to $1100^{\circ} \mathrm{C}$, the Raman intensity of both TO and LO phonon modes becomes comparable. It suggests that the InAs QDs are randomly oriented. Also the TO and LO phonon modes peak position recorded from the samples containing GaAs or InP nanocrystals are in good agreement with the value for a bulk crystal [17].

Moreover, the Raman spectra obtained from the samples containing $\mathrm{Ga}$ and As annealed at different temperatures in the range $1100-1350^{\circ} \mathrm{C}$ reveals different intensity ratios between the TO and LO phonon modes (not shown here). After annealing up to $1200^{\circ} \mathrm{C}$ both phonon modes have similar intensities, which means that GaAs QDs are randomly oriented. When the annealing temperature increases up to $1300^{\circ} \mathrm{C}$, the LO phonon mode dominates indicating that most of the GaAs QDs are oriented along (100) plane. An increase of the annealing temperature above $1300{ }^{\circ} \mathrm{C}$ leads to (111)-oriented GaAs QDs formation.

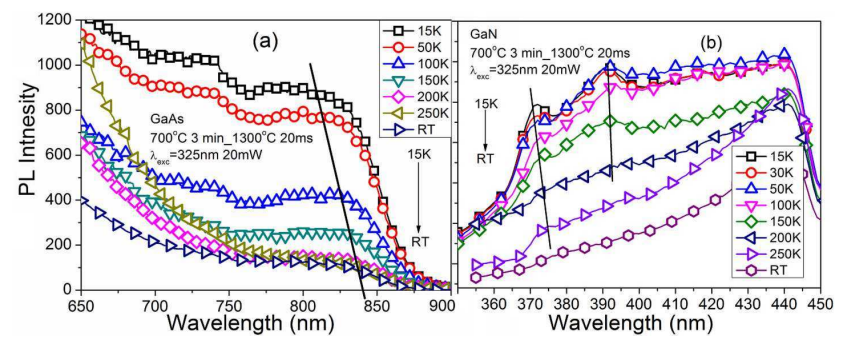

Fig. 3. TDPL spectra obtained from GaAs QDs (a) and $\mathrm{GaN}$ QDs (b) embedded in the $\mathrm{SiO}_{2}$ layer during UV (325 nm) laser excitation.

The TDPL was performed on the samples containing GaAs or GaN QDs in the $\mathrm{SiO}_{2}$ layer in the temperature range $15-300 \mathrm{~K}$ during laser excitation at $325 \mathrm{~nm}$ wavelength with $20 \mathrm{~mW}$ power (see Fig. 3). At low temperature typical luminescence corresponding to the GaAs and GaN nanocrystals was found. The nanocrystals related PL peak position at $15 \mathrm{~K}$ is located at around 825 and $370 \mathrm{~nm}$ for GaAs and GaN, respectively. It matches well with the band gap of bulk GaAs and GaN [18]. Moreover, with the increase of the measurements temperature, the nanocrystals related luminescence exhibited typical redshift which further confirms the origin of luminescence. In the case of the sample containing GaN QDs, another band at around $395 \mathrm{~nm}$ was observed. This lumines- 
cence shows only the slight red shift with the increasing temperature which is typical of the defect luminescence centers. The PL peak at $395 \mathrm{~nm}$ corresponds probably to the defects states in the $\mathrm{SiO}_{2}$ layer which was used in these experiments.

One of the main defect centers in the oxide layer is the oxygen deficient center (ODC). The ODC related luminescence is usually located at around 440-480 nm [19]. The energy levels of ODC are strongly affected by surrounded atoms. Germanium implanted oxide shows the ODC related luminescence at around $395 \mathrm{~nm}$ [20]. Hence, gallium implantation may have a similar effect. Another explanation for the $395 \mathrm{~nm}$ emission can be linked to $\beta-\mathrm{Ga}_{2} \mathrm{O}_{3}$ which can be formed in the oxide layer as well. Since the matrix is composed of silicon and oxygen atoms, the GaN nanocrystals are surrounded by oxygen which forms the core-shell oxide layer around GaN QDs. The defects states in $\beta-\mathrm{Ga}_{2} \mathrm{O}_{3}$ can be optically populated and emit in the UV-blue spectrum range [21].

\section{Conclusions}

We have shown that different III-V compound semiconductors nanostructures can be efficiently synthesized in the silicon based dielectrics using sequential ion implantation and millisecond range flash lamp annealing. During the high temperature ultrashort annealing the outdiffusion of implanted elements is suppressed, which significantly reduces the minimum implantation fluence needed for the nanocrystals formation. The data obtained by microstructural and optical investigations of III-V QDs confirms high quality nanocrystals formation. The combination of ion implantation and FLA seems to be the most useful for such proposal. The presented systems containing QDs in dielectrics may find potential application in the optoelectronics and for memory devices.

\section{Acknowledgments}

This work was partially supported by the Polish Ministry of Science and Higher Education, grant No. N N515 246637 and the Helmholtz-Gemeinschaft Deutscher Forschungszentren (HGF-VH-NG-713).

\section{References}

[1] M. Hocevar, N. Baboux, A. Poncet, M. Gendry, A. Souifi, IEEE Trans. Electron. Dev. 56, 2657 (2009).

[2] D. Dvorak, B.L. Justus, D.K. Daskill, D.G. Hendershot, Appl. Phys. Lett. 66, 804 (1995).
[3] T. Shimizu-Iwayama, K. Fujita, S. Nakao, K. Saitoh, T. Fujita, N. Itoh, J. Appl. Phys. 75, 7779 (1994).

[4] R.L. Wells, S.R. Aubuchon, S.S. Kher, M.S. Lube, Chem. Mater. 7, 793 (1995).

[5] A. Meldrum, L.A. Boatner, C.W. White, Nucl. Instrum. Methods Phys. Res. B 178, 7 (2001).

[6] S. Prucnal, M. Turek, A. Drozdziel, K. Pyszniak, S.Q. Zhou, A. Kanjilal, W. Skorupa, J. Zuk, Appl. Phys. B 101, 315 (2010).

[7] S. Prucnal, M. Turek, A. Drozdziel, K. Pyszniak, A. Wójtowicz, S.-Q. Zhou, A. Kanjilal, A. Shalimov, W. Skorupa, J. Zuk, Cent. Eur. J. Phys. 9, 338 (2011).

[8] S. Prucnal, L. Rebohle, A. Kanjilal, H. Krzyzanowska, W. Skorupa, Electrochem. Solid-State Lett. 12, H333 (2009).

[9] R. Heitz, N.N. Ledentsov, D. Bimberg, A. Yu, M.V. Egorov, V.M. Maximo, A. Ustinov, E. Zhukov, Zh. I. Alferov, Appl. Phys. Lett. 74, 1701 (1999).

[10] S. Prucnal, S. Facsko, C. Baumgart, H. Schmidt, M.O. Liedke, L. Rebohle, A. Shalimov, H. Reuther, A. Kanjilal, A. Mücklich, M. Helm, J. Zuk, W. Skorupa, Nano Lett. 11, 2814 (2011).

[11] S. Prucnal, S.-Q. Zhou, X. Ou, H. Reuther, M.O. Liedke, A. Mücklich, M. Helm, J. Żuk, M. Turek, K. Pyszniak, W. Skorupa, Nanotechnology 23, 485204 (2012).

[12] M. Turek, S. Prucnal, A. Drozdziel, K. Pyszniak, Rev. Sci. Instrum. 80, 043304 (2009).

[13] M. Turek, S. Prucnal, A. Drozdziel, K. Pyszniak, Nucl. Instrum. Methods Phys. Res. B 269, 700 (2011).

[14] M. Turek, A. Droździel, K. Pyszniak, S. Prucnal, J. Zuk, Przeglad Elektrotechniczny 86, 193 (2010) (in Polish).

[15] A.L. Lin, L.F. Donaghey, J. Electr. Mater. 7, 383 (1977).

[16] G. Armelles, T. Utzmeier, P.A. Postigo, F. Briones, J.C. Ferrer, P. Peiró, A. Cornet, J. Appl. Phys. 81, 6339 (1997).

[17] A. Mooradian, G.B. Wright, Solid State Commun. 4, 431 (1966)

[18] T. Ando, A.B. Fowler, F. Stern, Rev. Mod. Phys 54, 437 (1982).

[19] S. Prucnal, J.M. Sun, L. Rebohle, W. Skorupa, Appl. Phys. Lett. 91, 181107 (2007).

[20] A.N. Nazarov, I.N. Osiyuk, J.M. Sun, R.A. Yankov, W. Skorupa, I.P. Tyagulskii, V.S. Lysenko, S. Prucnal, T. Gebel, L. Rebohle, Appl. Phys. B 87, 129 (2006).

[21] S.J. Xu, G. Li, S.J. Chua, X.C. Wang, W. Wang, Appl. Phys. Lett. 72, 2451 (1998). 